\title{
LA GOLETA: COMIENZOS DE UNA FORTALEZA
}

\author{
Por \\ JORGE VERA APARICI
}

\author{
Dedicatoria: \\ - A las autoriades tunecinas. \\ - Al Ministerio de Defensa Nacional Argelino. \\ - Al Agregado Militar en Argel, coronel Castillo Payán, \\ agradeciéndoles su colaboración.
}

Conocemos el aspecto de la Goleta en los primeros años del siglo XVI a partir de las noticias de fray Diego de Haedo y de Luis del Mármol (1): era fundamentalmente una pequeña torre de base cuadrada, construida sobre la orilla septentrional del canal que une el lago de Túnez con el mar, en la estrecha franja de tierra que separaba el «stagno» y ciudad de Túnez de un lado y el mar de otro, según una disposición similar al idealizado plano de Bertelli (2) «ll vero disegno della cittá di Tunisi, e Bizerta». El cometido de la torre era el de servir de aduana y control de mercancías que alli eran descargadas por las naves comerciales con destino a la capital del reino hafsida.

Desde que en 1516 Barbarroja se apoderó de Argel, la acción de aquél sobre diferentes puntos del litoral africano se dejó sentir con fuerza: en 1534 conquista la ciudad de Túnez y advertido de la importancia estratégica del canal, se dispone a

(1) «... llegó Aruch a Túnez tomando tierra en la Goleta, que entonces no era más que una torre pequeña, que servía de Aduana en que los navíos de mercaderes que por mar contrataban en Túnez, descargaban todas sus mercaderias: y fué esto en el verano del año de Nuestro Señor lésu Christo. 1504». DE HAEDO, FRAY DIEGO: Topofrafía e Historia general de Argel. Epitome de los reyes de Argel, Valladolid, 1612, libro II, cap. I, fol. 47; «Antes que Barbarroxa fortalesció la Goleta era un sola torre quadrada como casa de aduana puesta en la boca del canal donde entra el agua de la mar en el estaño que está delante de Túnez. Este canal es largo cuanto un tiro de ballesta y tan estrecho que no puede pasar una galera remando por él», MÁRMOL CARVAJAL, LUIS DEL:Descripción general de Africa, Granada 1573, lib. VI, fol. 250

(2) BERTELLI: «ll verso disegno della cittǎ di Tunisi, e Bizerta», Venecia, 1574. 
ampliar y reforzar la pequeña torre cuadrada existente.

Siguiendo a Diego de Haedo en su descripción de la fortaleza, afirma que Arūŷ Barbarroja transformó la débil torre que allí se encontraba en una buena y bella fortaleza bien armada y bien aprovisionada de municiones (3), de esta manera la torrecilla de vigilancia y control aduanero se convirtió definitivamente en fortaleza, añadiéndosele en sus cuatro ángulos exteriores otras tantas torres de base cuadrada con su correspondiente capacidad artillera (4). Este reforzamiento de la torre parece más verosímil y acorde con la documentación gráfica de la época y con el breve espacio de tiempo del que dispuso Barbarroja para realizarlo, que la fortificación «con bastiones y terraplenes muy fuertes» que describe Diego de Haedo. A esta ampliación fundamental Barbarroja añadirá, poco tiempo antes de la expedición militar de Carlos $V$ en el verano de 1535, una serie de fortificaciones anejas de menos importancia y de carácter temporal, como fue la muralla de tierra y troncos algo alejada de la fortaleza en dirección oeste, que albergó una importante batería de defensa durante el sitio efectuado por el ejército de Carlos V, como se desprende al observar la colección de tapices conservados en el Palacio Real de Madrid (5), tejidos según los cartones de Jean Vermeyen, que acompañaba la expedición militar sobre Túnez en calidad de cronista gráfico y artístico de la misma. El artista flamenco nos presenta una fortaleza con cinco torres cuadradas adosadas al cuerpo prismático central, una en cada vértice del mismo y la quinta reforzando la muralla recayente al mar, el acceso principal se había situado en el lado orientado al estanque. Poseía sólidos muros, una gran cisterna y almacenes para provisiones en sus recintos abovedados ordenados en dos plantas.

La fortaleza de la Goleta fue tomada en julio de 1535 por un ejército integrado por españoles, alemanes e italianos, después de sufrir un abundante fuego artillero, conquistando a continuación la ciudad de Túnez y su Alcazaba. Concluida la conquista y antes de partir la expedición militar, Carlos $V$ nombró a Bernardino de Mendoza gobernador de la plaza, dejándole al mando de 1.200 veteranos españoles (6), encargándole las obras de mejora y fortificación de la misma en colaboración con el almirante Andrea Doria, el virrey de Sicilia y el ingeniero militar Ferramolino. Desde el mismo Túnez dispuso asimismo el Emperador que los primeros materiales y aprovisionamientos necesarios para la construcción y mantenimiento de la fortaleza y su guarnición se enviasen desde Sicilia, realizándose el primer envío a la Goleta en septiembre de 1535 (7). Posteriormente, el aprovisionamiento desde Sicilia se combinaria con las posibilidades de materiales constructivos que el país ofrecía: se de-

(3) «Tras esto la primera cosa que hizo (Barbarroja). fué fortificar luego la Goleta, con bastiones, y terraplenos muy fuertes, con que de una torre muy pequeña, y flaca, la hizo una fuerça muy grande, muy principal, en la cual puso muy gran número de Artillería y municiones, y hasta mil y quientos turcos de guarnición. La cual obra acabó aquel invierno siguiente, trayendo muchos moros Alarbes gastadores de fuera que nunca cesaban de trabajar», HAEDO, FR. DIEGO DE, op cit., libro II, cap. II, fol. 58 .

(4) «En fait, Barberousee consolida la tour carrée qui se trouvait sur la rive septentrionale du canal et la flanqua aux quatre angles de bastions carrés». SEBAG, PAUL: «La Goulette et sa forteresse de la fin du XVI siècle à nos jours", en IBLA, Tunis, 30, 1967, pág. 14.

(5) JUNQUERA DE VEGA, P. y otros: Catálogo de tapices del Patrimonio Nacional, Madrid, 1986, 1 vol.

(6) MÁRMOL CARVAJAL, LUIS DEL, op. cit., lib. II, fol. 487.

(7) POINSSOT y LANTIER: "Les gouverneurs de la Goulette durant l'occupation espagnole (1535-1574)», en Re vue Tunisienne, Tunis, 1930, pàg. 223. 
molieron fragmentos del acueducto de Adriano para procurarse piedra, comerciándose la cal y el yeso con los habitantes de Túnez (8), como se indica en la carta de Francisco de Tovar al Emperador, fechada en abril de 1538, en la que afirma: «Piedra hay también en los arcos y toda tan buena y labrada como V.M. ha visto, el inconveniente es que no habrá parejo de traerse con tanta furia como habría menester porque son los moros los que la traen (y) el rey de Túnez no deja traer y no con carretas aunque los moros con buena gana traerían, con muchas yo he enviado a Nápoles por treinta carretas y aún pienso comprar bueyes que los hay a buen precio y hacer traer piedra de los arcos que esta paresce en la verdad la que más barata se puede traer y se ahorra mucha cal con esta porque (son) grandes más que otras y ahorran muchos oficiales (...)» (9).

Durante tres años se habían sucedido las obras que modificaron y consolidaron lo construido por Barbarroja, eliminando parte de las torres adosadas y emprendiendo los trabajos de ampliación de la fortaleza. La nueva planta de la misma es concebida por Bernardino de Mendoza, remitiéndose al Emperador el primer documento gráfico conocido de esta construcción militar; el dibujo fue realizado por una mano inexperta y Francisco de Tovar, futuro segundo gobernador de la Goleta, lo califica como la mejor traza respecto del otro plano realizado por el virrey de Sicilia, documento hasta el momento desconocido (10).

El dibujo remitido desde Túnez el 30 de abril de 1538 ilustraba la solución propuesta por Mendoza, consistente en una planta del recinto interior en forma de trapecio rectángulo, en cuyos vértices se situaban tres baluartes de nueva fábrica y en el cuarto vértice se conserva la torre conquistada a Barbarroja. En las formas escogidas para los baluartes se observan importantes diferencias. El baluarte próximo al mar, de planta pentagonal, no coincide con los diseños más frecuentes de los baluartes de la época, se representa con dos piezas de artillería y próximo al baluarte de planta triangular, que observa en parte los criterios de regularidad geométrica vigentes en la arquitectura militar del momento y de utilización de formas en punta de flecha. El que se representa con una única pieza de artilleria es en realidad un semibastión, prologándose uno de sus lados hasta encontrar perpendicularmente la muralla con la puerta de acceso de la antigua torre de Barbarroja, que se aprovecha como factor importante en la defensa del acceso al canal desde el mar.

En la carta que acompañaba el mencionado plano se afirma que los muros están aún bajos por la parte del mar, que la batería que el mismo Emperador había mandado hacer está sin foso, ya que a pesar de haberlo excavado en diferentes ocasiones los vientos habian arrastrado suficiente arena para cegarlo. Se solicitaban asimismo canteros que sepan trabajar la piedra y que provengan de España (11), señalando la necesidad de escoltas para realizar el aprovisionamiento y transporte de piedra a causa de los hostigamientos de los naturales del país.

(8) Ibidem.

(9) Carta de D. Francisco de Tovar al Emperacior sobre la fortificación y defensa de la Goleta y otros asuntos. La Goleta, 30 de abril de 1538, Archivo General de Simancas, Guerra Antigua, leg. 13, fol. 91.

(10) Carta de D. Francisco de Tovar al Emperador. La Goleta, mayo de 1538, Archivo General de Simancas, Guerra Antigua, leg. 13, fol. 96.

(11) Archivo General de Simancas, Guerra Antigua, leg. 13, fol. 125. 
Para 1538 estaban construidos al menos dos de los tres nuevos bastiones propuestos en el plano de Bernardino de Mendoza. La arena que se sacaba de los fosos no era posible utilizarla para formar la necesaria tierra compactada que constituía el «terrapleno» del baluarte, que se recubría de sólidos muros de sillería.

En mayo de 1538 Francisco de Tovar aconseja al Emperador que «(..) debería de venir de una vez, quince o veinte naves con piedra y cal (...)» (12). Este cambio de opinión respecto de la provisión de materiales constructivos podría haber sido provocado por el deseo de eludir el obligado pago a los que comerciaban con la cal y el yeso, o por las continuas agresiones de los enemigos, que obligaban a crear importantes escoltas para la extracción y transporte de piedras.

En cuanto a la disposición irregular y asimétrica del primer plano conocido del fuerte de la Goleta, pudo ser debida a un intento de adecuación al terreno sobre el que se asentaba, ya que si consideramos los diseños de Vermeyen veraces en cuanto a la disposición de los principales accidentes geográficos, estudiados por Monchicourt (13), podremos afirmar que a la llegada de los españoles a la Goleta existía al final del canal, por el lado del estanque y de la torre fortificada, una lengua de tierra que se adentraba en el estanque en dirección a Túnez, cuyo tamaño permitió la construcción del semibastión con una sola pieza de artillería. La ensenada que a partir de esa lengua de tierra formaría el contorno del lago, obligaría a diseñar una muralla oblicua respecto de la línea recta torre de Barbarroja-semibastión, y no perpendicular a ella, como hubiera sido lo obligado según los criterios de racionalidad y regularidad geométrica utilizados por la arquitectura militar renacentista, defensora de los preceptos de la simetría para el desarrollo de las plantas de sus edificaciones.

Los trabajos de construcción fueron especialmente duros, dadas las condiciones de mal alojamiento de la tropa, de su necesidad de defenderse de ataques continuos, así como por la falta constante de víveres y por las condiciones adversas del terreno, dada su humedad constante y la incidencia continua de la arena que cegaba los pozos y fosos.

Posteriores al primer plano de la Goleta se conocen tres dibujos en planta de! ingeniero Ferramolino de sumo interés para conocer la evolución de la fortaleza. El primero de ellos es el titulado «El primo desegno del fuerte de la Goleta» (14), acompañaba una carta de Ferramolino escrita el primero de agosto de 1539, quince meses después de ser enviado el proyecto defendido por Bernardino de Mendoza. Las diferencias entre ambos son elocuentes. Ferramolino opta por un modelo inspirado en la regularidad geométrica: planta triangular, con dos baluartes idénticos con sus respectivos caballeros en dos de sus vértices y en el tercero aprovechamiento parcial de la torre de Barbarroja a la que se adiciona un semibastión con una batería de seis piezas, un pequeño revellín independiente se sitúa al otro lado del canal, al que se accede desde la muralla de la propia fortaleza. Todo el conjunto está rodeado de un foso y otros «reparos» secundarios y exteriores. Tanto la triangularidad de

(12) Carta de D. Francisco de Tovar al Emperador, Archivo General de Simancas, Guerra Antigua, leg. 13 , fol. 95.

(13) MONCHICOURT, CH.: «Essai bibliographique sur les plans imprimés de Tripoli, Djerba et Tunis-Goulette au $\mathrm{XV}$ l e siècle», en Revue Africaine, Alger, 1925.

(14) Archivo General de Simancas, mapas, planos y dibujos, XIX-104. 
«El segondo disegno» del fuerte de la Goleta (16) acompañaba la misma carta y plano del ingeniero militar italiano citados anteriormente y suponía un cambio fundamental respecto del «Primo desegno", constituyendo el perfeccionamiento de la idea primigenia de Mendoza, del que se conserva la antigua torre de Barbarroja a la que se agrega un reducido bastión orientado al mar, se conserva asimismo la situación del baluarte orientado al lago y la ciudad de Túnez, mejorándolo y ampliándolo, y se orientan los dos baluartes restantes hacia la antigua Cartago, defendiendo la planta, la situación de caballeros, creación de un revellín exterior que cubre la cortina del canal (aportación de la arquitectura militar de finales del siglo XV que se desarrollará ampliamente a lo largo del siglo XVIII), son plenamente renacentistas y contrastan con el primer dibujo conocido de la Goleta, vacilante y aún pobre en conocimientos del arte militar de fortificación. La potente capacidad de fuego (68 bocas de fuego al exterior), la previsión de murallas complementarias exteriores y el caballero o torre octogonal central conforman un conjunto debidamente estructurado, cerrado y supuestamente reducido, ya que no se señala escala alguna ni especifica almacenes, cisternas o alojamientos de la tropa, percibiéndose cierto apresuramiento en la realización de este proyecto.

La concepción de un fuerte de tamaño reducido para la Goleta, en contraposición a la de una fortaleza más extensa y compleja, obedecía a razones de índole económica en lo tocante a la construcción y mantenimiento del lugar fortificado. Ferramolino afirmaba (15) que se realizaba grandísimo gasto en la construcción del tipo de fortaleza elegido para la Goleta, basándose en idéntica opinión del príncipe Andrea Doria y que con la fortaleza de planta triangular por él propuesta hubieran sido necesarios sólo 400 soldados para guardarla y treinta y cinco mil ducados para su construcción. El mismo virrey de Sicilia apoya este proyecto simplificado de fortaleza, pero los ánimos del Emperador se decantan por escuchar la opinión de Francisco de Tovar que habia apoyado incondicionalmente el proyecto más costoso de Bernardino de Mendoza, que coincidia con las pretensiones cesáreas de otorgar a la Goleta la función de llave del reino de Túnez, asegurando así la victoria conseguida al poseer un potente aparato coercitivo y de control sobre el acceso a Túnez por mar. La importancia estratégica de esta fortaleza no pasaría desapercibida ante el Emperador o sus jefes militares, ya que el arco defensivo constituido por las fortalezas que jalonaban el norte de África desde Melilla hasta Tripoli, protegiendo el flanco sur de la Península Ibérica o ltálica, entraba en contacto con el arco defensivo constituido por las fortificaciones del reino de Nápoles y Sicilia (fachadas orientales principalmente) e isla de Malta, que actuaban como «bisagra» o cierre y contención en la zona intermedia y más estrecha del Mediterráneo. La Goleta jugaba así un papel estratégico-fundamental como nexo de unión entre ambos.

La solución propuesta por el ingeniero Ferramolino, que eliminaba el bastión más cercano al mar del primer proyecto, además de otras innovaciones importantes, después de más de un año de comenzadas las obras a partir de la primera idea original, nos permite deducir un desarrollo incipiente de las obras, aunque noticias anteriores dan por acabados dos de los tres bastiones originalmente propuestos.

(15) Carta de Ferramolino al Rey, Archivo General de Simancas, Guerra Antigua, leg. 14, fol. 47

(16) Archivo General de Simancas, mapas, planos y dibujos, XIX-105. 
el acceso por tierra y unidos por una muralla de dirección quebrada, rodeado todo ello por un foso. Del «Primo disegno» se conserva la torre artillada octogonal, centro geométrico del conjunto y el revellín aislado del otro lado del canal. Existe una homogeneización de los distintos baluartes, aprovechamiento de los restos defensivos anteriores y adaptación a las fronteras naturales impuestas por el mar al norte, el canal al este y el lago al sur. No se proporcionan escalas o medidas acotadas, concediendo 83 bocas de fuego al exterior. Esta concepción de la fortaleza supondría, según Ferramolino, un gasto para la Corona de cincuenta y cinco mil ducados para su construcción y una guarnición aproximada de 500 soldados.

La cuestión del estado de las obras de la fortaleza en agosto de 1539, independiente de los aspectos proyectuales mencionados, se puede analizar a partir del tercer plano remitido por Ferramolino y titulado «Como sta la Goleta al presente» (17). El dibujo, en planta de trapecio rectángulo, posee baluartes diferenciados en sus cuatro vértices; de los cuales, los dos más cercanos al lago poseen la característica esctructura abaluartada de la época, con sus respectivos caballeros; mientras que los dos próximos al mar suponen, el primero, futuro baluarte de Santa Bárbara, una tenaza adosada a la antigua torre de Barbarroja (de planta rectangular después de su ampliación), el segundo, futuro baluarte de San Jorge, es un bastión reducido con dos dimensiones alternativas: una estampada sobre el propio papel en el que se dibuja el plano y otra dibujada sobre un fragmento de papel pegado al anterior.

El conjunto defensivo respeta en gran medida «El secondo desegno», únicamente el futuro bastión de San Jorge, de dimensiones muy reducidas, está aún inacabado, al igual que la torre octogonal central a la que no se hace ninguna referencia, la muralla de dirección quebrada se sustituye por una cortina de dirección rectilínea

Las modificaciones posteriores respecto del plano «Como sta la Goleta al presente" van a ser notables, ya que la planta trapezoidal se transformará en rectangular, los baluartes de San Jorge y Santa Bárbara serán profundamente modificados en su estructura y lo que es más sorprendente, el baluarte orientado hacia Cartago cambiará de ubicación adentrándose en dirección hacia el lago hasta llegar a la altura del baluarte de San Miguel, que vigila la salida del canal al estanque; en esa nueva posición se le reconoce posteriormente y se le nombra como baluarte de Santiago. El de San Miguel será el único cuya situación y estructura no cambiará Ferramolino.

Durante los años treinta y posteriores los envíos de bastimentos fueron continuos, muchos de ellos procedian de Palermo; su flujo constante permitió que las obras avanzasen hasta ir constituyendo, no sin dificultades, la que se conoce como «Goleta Vieja». Materiales como tablas de roble, madera de clavazón, clavos, martillos, tenazas, azadones, hierro para forja, cáñamo, cal, piedra, brea, plomo, picos, astides, etc., las relaciones de materiales que se solicitan y se proveen son tan numerosas como extensas. Como muestra de esa actividad se ofrece un fragmento del «Sumario de lo que ha cargado la nave del capitán Balderi Marres que es de portadura 1.500 salmas que hizo vela del puerto de Palermo a veinte de mayo 1536 y ha de tomar el cumplimiento de la cargación en Trapana para llevarlo a la fortaleza de la Goleta (18):

(17) Archivo General de Simancas, mapas, planos y dibujos, XIX-106.

(18) Archive d'Outre Mer (Aix-en-Provence), Gouvernement General de l'Algerie, serie C, p. 444 (fol. 128). 
tablas dos mil, cien salmas de carbón de herreria, veinticinco tablones de olmo para carretas, ochenta y tres troncos de roble, una culebrina con sus ruedas y aforo, cincuenta pelotas, dos esmeriles, etc.».

Si la Goleta Vieja -con sus cuatro bastiones principales ya mencionados- se construyó durante el reinado de Carlos V, la Goleta Nueva corresponde principalmente al reinado de Felipe II. Concebida por el arquitecto militar II Fratino como una fortaleza extensa y costosa con doce baluartes y revellines, llegó a englobar a la primitiva fortificación a modo de una potente primera línea fortificada. Su complejidad y aparatosidad (llegó a albergar 500 piezas de artillería) le otorgó fama de inexpugnable que perderá al ser conquistada por los turcos en 1574. Tal volumen construido exigía enormes gastos de mantenimiento en hombres, municiones, provisiones y materiales constructivos, Fernand Braudel señala un gasto para obras en la Goleta de 50.000 ducados en 1566 y otros 50.000 dos años más tarde, sin contar otros posibles en ese período (19).

(19) BRAUDEL, FERNAND: El Mediterráneo y el mundo mediterráneo en la época de Felipe ll, México, 1953, vol. II, pág. 275 . 